\title{
MILLISECOND PULSAR FORMATION AND EVOLUTION
}

\author{
E.P.J. van den Heuve1 \\ Astronomical Institute \\ University of Amsterdam \\ Roetersstraat 15 \\ 1018 WB Amsterdam, The Netherlands
}

\begin{abstract}
The evolutionary history of binary radio pulsars, including the two millisecond binary pulsars, is reviewed. There are two groups of binary pulsars, the PSR 1913+16-group, which descended from massive X-ray binaries, and the PSR 1953+29-group, which descended from fairly wide low-mass $X$-ray binaries. The neutron stars in the second group probably formed by the accretion-induced collapse of a massive white dwarf. The companion stars in both groups of systems are expected to be dead stars, i.e. white dwarfs or neutron stars.

The large total number of millisecond binary pulsars in the galaxy $\left(\sim 10^{4}\right)$, indicates that magnetic fields of neutron stars do not decay below a value of order $10^{9} \mathrm{G}$. Possible explanations for this phenomenon are discussed.

Coalescence with a close degenerate companion provides a viable model for the formation of the single millisecond pulsar.
\end{abstract}

\section{INTRODUCTION}

Three millisecond pulsars are known, two of which are in binary systems. With the other binary radio pulsars, they have in common (see table 1):

- an unusually rapid rotation: three out of the five most rapidly spinning pulsars known are in binaries, while in total only seven out of the about 500 known pulsars are in binaries.

- an unusually weak surface dipole magnetic field; figure 1 depicts this clearly: only 2 out of the 7 binary pulsars have magnetic field strengths in the same range as that of the bulk of the single pulsars.

The two binary millisecond pulsars thus appear to form the short-period portion of the pulse period distribution of the general binary pulsar population. Their evolutionary history is therefore expected to have been similar to that of the other binary pulsars.

The single millisecond pulsar PSR $1937+21$ is a different case but, as we will argue in the last section, also its origin is likely to be tied with the evolution of binary systems.

For detailed reviews of the evolutionary history of binary and 
millisecond radio pulsars and X-ray binaries we refer to Van den Heuvel (1984, 1986a, b), Van den Heuvel and Habets (1985) and Taam and Van den Heuvel (1986). Here we only summarize the most important recent developments.

TABLE 1. Observed and derived parameters of binary and millisecond pulsars. The maximum ages correspond to spindown at constant B from the spin-up line up to the present spin period. The times till turn off are the times required for reaching the deathline by spindown at constant B. For PSR $1913+16$ the latter time is much longer than the coalescence time of the system $\left(\sim 3.10^{8}\right.$ yrs). PSR $1831-00$ is assumed to be situated above the deathline (see figure 1). (Observed parameters from Dewey et al. 1986).

\begin{tabular}{|c|c|c|c|c|c|c|c|c|c|}
\hline PSR & $\begin{array}{c}P \\
\text { (ms) }\end{array}$ & $\log \dot{\mathrm{P}}$ & $\log _{(G)} B$ & $\begin{array}{c}P_{b} \\
\text { (days) }\end{array}$ & e & $\begin{array}{c}\mathrm{f}\left(\mathrm{m}_{1}, \mathrm{~m}_{2}\right) \\
\left(\mathrm{M}_{\odot}\right)\end{array}$ & $\begin{array}{c}\text { Likely } \\
\left(M_{\odot}\right)\end{array}$ & $\begin{array}{l}\text { Max. } \\
\text { Age } \\
\left(10^{9} \text { yr) }\right.\end{array}$ & $\begin{array}{l}\text { Time till } \\
\text { turn-off } \\
\left(10^{9} \mathrm{yr}\right) \\
\end{array}$ \\
\hline $37+21$ & 1.6 & -19.0 & 8.65 & --- & --- & -- & -- & 0.1 & $>t_{\mathrm{Hub}}$ \\
\hline & & -19.5 & 8.6 & 117.35 & 0.0003 & 0.0027 & $0.2-0.4$ & 2.9 & \\
\hline & 5. & -19.4 & 8.7 & 12. & 0.0 & & $0.2-0.4$ & 2 & $>t_{\mathrm{Hub}}$ \\
\hline & & & & & & & $0.7-1.3$ & & 5.3 \\
\hline & 59 & -17.1 & 10. & 0.32 & 0.6171 & 0.1 & 1.4 & 0.0 & 4.2 \\
\hline & & $<-17.0$ & $<10$ & & $<0.0$ & & $0.06-0.13$ & -2.1 & $<0.3$ \\
\hline & 864.9 & -16.0 & & 1232.40 & 0.01 & 0.0 & $0.2-0.4$ & 0.1 & 0.2 \\
\hline $2303+46$ & 1066.4 & -15.4 & 11.8 & 12.34 & 0.6584 & 0.2463 & $1.2-2.5$ & 0.033 & 0.08 \\
\hline
\end{tabular}

2. THE BINARY "RECYCLING" MODEL FOR THE ORIGIN OF BINARY RADIO PULSARS.

2.1. Period and magnetic field evolution of newborn neutron stars

Statistical investigations of radio pulsars show that the quantity ( $\dot{P}$ ) decreases in the course of time on a timescale of order $5.10^{6} \mathrm{yrs}$ (cf. Lyne, this volume). This quantity is related to the surface dipole magnetic field of the neutron star by the relation (cf. Flowers and Ruderman 1977)

$$
(P \cdot \dot{P})=K\left(B_{\perp}^{2}+\xi B_{/ /}^{2}\right)
$$

where $B_{\perp}$ is the component of the magnetic field perpendicular to the rotation axis, $\mathrm{B}_{/ /}$is the component parallel to the rotation axis, and $\mathrm{K}$ is a constant which contains the moment of inertia of the neutron star. The quantity $\xi$ is related to the charge flow in the magnetosphere of the neutron star. Its expected value depends on the adopted model for the emission of the radio pulses. Ruderman and Sutherland (1976) estimate $\xi$ to be close to unity, but there are also models with a much lower value of $\xi$ (cf. Arons 1981).

We will, for the sake of argument, adopt with Ruderman and Sutherland that $\xi \simeq 1$. In that case $(P \cdot P)$ is a measure of the absolute value of the magnetic field strength. The magnetic field strengths in figure 1 were derived in this way, by adopting a standard moment of 
inertia of neutron stars of $10^{45} \mathrm{~g} \cdot \mathrm{cm}^{2}$ (for details: see Radhakrishnan 1982, 1986; van den Heuvẹl 1984).

The decrease of $(P . \dot{P})$ in the course of time implies, in terms of this model, that the surface dipole magnetic field strength decays on a timescale of $10^{6}-10^{7}$ yrs.

The dashed tracks in figure 1 indicate how a pulsar will evolve through the B-P diagram when its field decays on a timescale of $2.10^{6}$ yrs. When the pulsar passes a "deathline" the emission of pulsed radiation ceases and the pulsar enters the "graveyard", which is filled with extinct pulsars. The deathline drawn in figure 1 is that given by the Ruderman-Sutherland model.
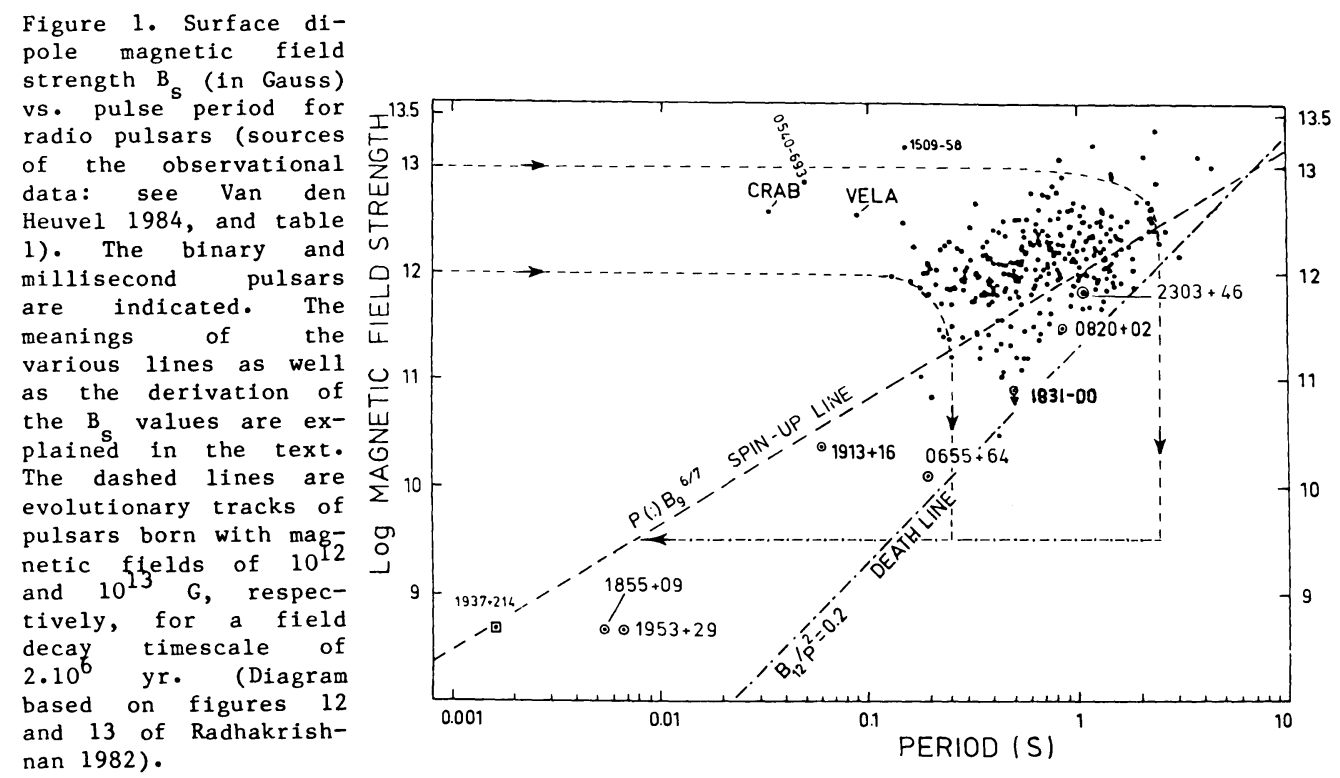

\subsection{Resurrection from the graveyard}

For single pulsars there is no way back from the graveyard. However, when a neutron star is in a binary system and its companion begins to transfer matter to it, its rotation will be accelerated due to the angular momentum carried with the transferred matter. This is clear from the gradual spin-up observed for all neutron stars in X-ray binaries which are fed by disk-accretion (cf. Rappaport and Joss 1983).

When it is being spun up the neutron star may already be quite old such that its magnetic field may have partially decayed. The dash-dotted horizontal line in figure 1 depicts schematically how an old neutron star in a binary may be spun-up to the region of the "living" radio pulsars once its companion evolves away from the main-sequence and begins to transfer matter to it (cf. Bisnovaty-Kogan and Komberg 1974; Smarr and Blandford 1976).

When the gas density around the neutron star has become very low, 
such that the radio signal will not be dispersed beyond recognition, the neutron star may become observable as a radio pulsar. This requires that also its companion must have terminated its life, and has become a compact object, either a neutron star, a white dwarf or a black hole.

2.3. The spin-up line

The shortest possible spin period that can be reached by accretion is the equilibrium spin period $P_{\text {eq }}$ (for the definition: see e.g: Henrichs 1983), corresponding to the maximum possible accretion rate $\dot{M}_{\text {edd }} \simeq 1.5 \times$ $10^{-8} \mathrm{M}_{\odot} / \mathrm{yr} \cdot \mathrm{P}_{\text {eq }}$ is given by (see Van den Heuvel 1977):

$$
\mathrm{P}_{\mathrm{eq}}=(1.9 \mathrm{~ms})\left(\mathrm{B}_{9}\right)^{6 / 7} \mathrm{M}^{-5 / 7}\left(\dot{\mathrm{M}} / \dot{\mathrm{M}}_{\mathrm{Edd}}\right)^{-3 / 7} \mathrm{R}_{6}{ }^{18 / 7}
$$

where $B_{9}$ is the surface dipole field strength in units of $10^{9} \mathrm{G}, \dot{M}$ is the accretion rate, and $M$ and $R$ are the mass and radius of the neutron star, in units of $1.4 \mathrm{M}_{\odot}$ and $10^{6} \mathrm{~cm}$, respectively. Adopting the latter two quantities to be unity, one observes that $\mathrm{P}_{\mathrm{eq}}$ is only a function of $\dot{\mathrm{B}}_{9}$ and $\dot{\mathrm{M}}$. Hence, the shortest attainable value of $\mathrm{P}_{\mathrm{eq}}$, which occurs for $\dot{\mathrm{M}}^{9}=\dot{\mathrm{M}}_{\mathrm{Edd}}$, is only a function of $\mathrm{B}_{9}$ :

$$
\mathrm{P}_{\text {eq, min }}=(1.9 \mathrm{~ms}) \mathrm{B}_{9} 6 / 7
$$

This relation defines the "spin-up" line, which is indicated in figure 1. All "recycled" pulsars are expected to be found in the wedge-shaped region between this line and the deathline. As figure 1 shows, all the 7 binary radio pulsars, as well as the single millisecond pulsar PSR1937+21 are indeed located in this region. This strongly suggests that they are recycled old pulsars.

2.4. The Evolutionary Status of the Companions of the binary radio pulsars

The fact that these pulsars are recycled at the same time, according to the above, implies that their companions themselves must now also be "dead" stars.

This expectation is confirmed in the cases of PSR $0655+64$ and PSR $0820+02$ by their optical identification (Kulkarni 1986a,b) which shows that the companions are located below the main sequence, in the HRdiagram, i.e.: are white dwarfs.

\subsection{The production of millisecond pulsars}

Figure 1 shows that in order to reach $\mathrm{P}<10 \mathrm{~ms}$, B should at the time of the spin up be smaller than $6 \cdot 10^{9} \mathrm{G}$. At larger field strengths, millisecond periods cannot be reached. A further constraint is that enough mass should be transferred, since otherwise not enough angular momentum can be transferred to achieve spin-up to a short period. For example, to reach $P=1.55 \mathrm{~ms}$ at $B_{S}=5.10^{8} \mathrm{G}$ requires an amount of accretion of at least $0.12 \mathrm{M}_{\odot}$. 


\section{THE TWO TYPES OF BINARY RADIO PULSARS AND THEIR ORIGIN}

\subsection{Introduction}

The 7 binary radio pulsars can be divided into two distinct classes, related to the two broad classes of binary $X$-ray sources, as follows (see table 1):

a. The "PSR 1913+16 class".

These are the three systems PSR 1913+16, PSR 2303+46 and PSR 0655+64, which have (i) relatively short orbital periods, (ii) relatively high companion masses $\left[\sim 1.4 \mathrm{M}_{\odot}\right.$ in PSR 1913+16 and PSR 2303+46, and $\sim 1 \mathrm{M}_{\odot}$ in PSR 0655+64], and (iii) very eccentric orbits in two out of three cases.

b. The "PSR $1953+29$ class".

These are the four other systems, which have:

(i) relatively long orbital periods, of order of weeks to years (the only exception is PSR 1831+00), (ii) very low mass functions,

indicating companion masses of $\sim 0.2-0.4 \mathrm{M}_{\odot^{\circ}}$ (iii) circular orbits.

The existence of these two classes can be understood on the basis of an evolutionary history with "spiral-in" for the first group, and "spiralout" for the second group (van den Heuvel and Taam 1984), relating them to the Massive $\mathrm{X}$-ray binaries (MXBs) and the Low-mass $\mathrm{X}$-ray binaries (LMXBs), respectively.

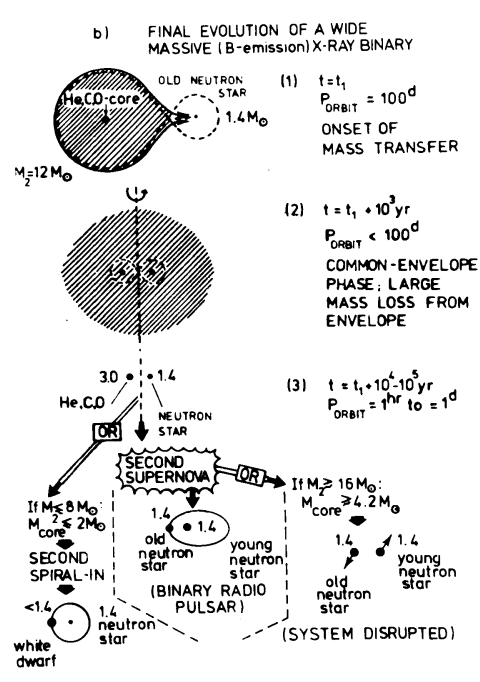

Figure 2a. Three possibilities for the final evolution of a massive X-ray binary into a radio pulsar binary or two runaway pulsars (explanation in section 3.2.).

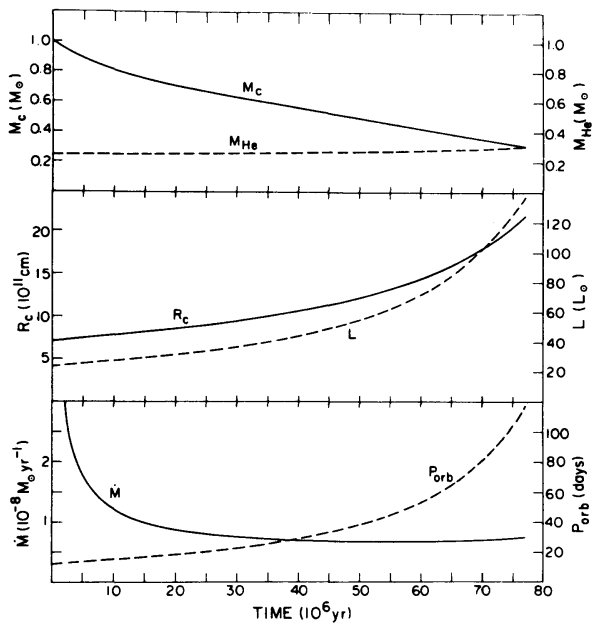

Figure 2b. Evolution of a wide lowmass $X$-ray binary into a radio pulsar binary of the PSR 1953+29type, as calculated by Joss and Rappaport (1983). The system started out with a $1 \mathrm{M}$ lower giant-branch star with a $1.3 \mathrm{M}_{\odot}$ neutron star companion in a 12.5 day orbit. Shown are the evolution of the companion mass $M_{C}$, its helium core mass $M_{H e}$, its radius $R_{C}$, luminosity $L$, mass transfer rate $\dot{M}$ and orbital period. Explanation in the text. 
Figures $2 \mathrm{a}$ and $\mathrm{b}$ depict the evolution in these two cases. Basically, the difference between the evolution of these two types of systems is due to the fact that, as a consequence of conservation of orbital angular momentum, transfer of mass from a more massive companion to the neutron star causes the orbit to shrink, whereas transfer of mass from a less massive companion causes the orbit to widen (see, e.g. Paczynski 1971). We will assume here that in both cases, the mass transfer is driven by the envelope expansion caused by the interior nuclear evolution of the star. We will now consider the two cases depicted in figure 2 in more detail.

\subsection{Origin of the "PSR $1913+16$ group"}

If the companion to a neutron star has a mass considerably larger than that of the neutron star, like in the MXBs, one expects that once the companion overflows its Roche lobe and begins to transfer matter to the neutron star, the neutron star will soon be entirely engulfed by the envelope of its companion. It will then rapidly spiral down into this envelope, as a consequence of the large frictional drag excerted on its orbital motion (see Taam et al. 1978; Bodenheimer and Taam 1985; Meyer and Meyer-Hofmeister 1979; Van den Heuvel 1986b). The expected outcome of the spiral-in is a very close binary system, consisting of the heavyelement core (He, and/or $\mathrm{C}$, 0 , etc.) of the companion together with the neutron star. Due to the frictional drag the orbit of this narrow system will be perfectly circular. For the further evolution of this narrow system there are three possibilities, depending on the core mass, as depicted in figure 2a (see also Habets 1985, 1986; Van den Heuvel and Habets 1985; Van den Heuvel 1986b):

(i) If $M_{\text {core }}<2-2.5 M_{\odot}\left(M_{\text {initial }}<8-10 M_{\odot}\right)$ a narrow system results consisting of a $\mathrm{C}-\odot$ white dwarf and the old "recycled" neutron star, in a circular orbit. PSR $0655+64$ presumably formed in this way.

(ii) For $2.5 M_{\odot}<M_{\text {core }}<4.2 M_{\odot}$ ( $M_{\text {initial }}$ in the range 10-15 $\left.M_{\odot}\right)$ the heavy-element core explodes as a supernova.

If there are no asymmetries in the explosion the system will not be disrupted and will consist of two neutron stars - one of them "recycled" - in an eccentric orbit. PSR 1913+16 and PSR 2303+46 are thought to have formed in this way (Flannery and van den Heuvel 1975; Smarr and Blandford 1976).

(iii) $M_{\text {core }}>4.2 M_{\odot}\left(M_{\text {initial }}>15 M_{\odot}\right)$. These systems are not expected to undergo further mass transfer before the core explodes as a SN. Again, adopting a circular orbit, a neutron star mass of $1.4 \mathrm{M}_{\odot}$ (for both stars) and no asymmetries, the system will be disrupted, since more than half of the system mass is ejected (Blaauw 1961). The result is: two runaway pulsars, one young and one old ("recycled").

3.3. Origin of the "PSR 1953+29-group" of binary pulsars

Figure 3 depicts the orbital dimensions and periods of 16 LMXBs with 
detected orbital motion. In the short-period LMXBs ( $\mathrm{P}_{\text {orb }} \leqslant 12^{\mathrm{h}}$ ) the mass transfer can be driven by gravitational radiation (GR) losses and magnetic braking (cf. Rappaport et al. 1983). On the other hand, in the wider systems such as Sco X-1, Cyg X-2, 2S0921-63 and GX 1+4 the mass transfer must be driven by the interior nuclear evolution of the companion star (Webbink et al. 1983; Taam 1983). This mechanism, moreover $\frac{1}{38}$ the only one that can explain the high X-ray luminosities of $\sim 10^{38} \mathrm{ergs} / \mathrm{s}$ of the about one dozen of strong bulge X-ray sources (Webbink et al. 1983). The mass-transfer rates in this case appear to be, throughout the evolution, given by simple formulae of the type (for $\mathrm{X}=0.70, \mathrm{Z}=0.02)$ :

$$
\dot{\mathrm{M}}=-6 \times 10^{-10} \mathrm{P}_{\mathrm{o}}(\mathrm{d}) \mathrm{M}_{\odot} / \mathrm{yr}
$$

where $P$ is the initial orbital period in days (this formula holds for $\left.\mathrm{P}_{\mathrm{O}}>2 \mathrm{~d}\right)$. Fig. 2b depicts the evolution of a system with $\mathrm{P}_{\mathrm{O}}=12.5$ as calculated by Joss and Rappaport (1983). The figure shows that at the end of the evolution only the helium core of the companion is left, with mass $M_{c}=0.31 M_{\odot}$, in an orbit with $P=117^{d}$, $i . e$. exactly reproducing the system parameters of PSR 1953+29. This model explains in a natural way two most important characteristics of the PSR $1953+29-$ group of systems :

(i) the low mass of the companion star, which is always the remnant helium core of $0.2-0.4 \mathrm{M}_{\odot}$ of the solar-type companion star.

(ii) the circularity of the orbits, since during the long time interval that the low-mass giant filled its Roche-lobe, the tidal

interaction in the systems was very strong.

It thus appears that the PSR $1953+29$-group of systems are the natural descendants of the wide LMXBs such as Cyg X-2, 2S0921-63, Sco X-1, GX $1+4$ and the bright bulge sources.

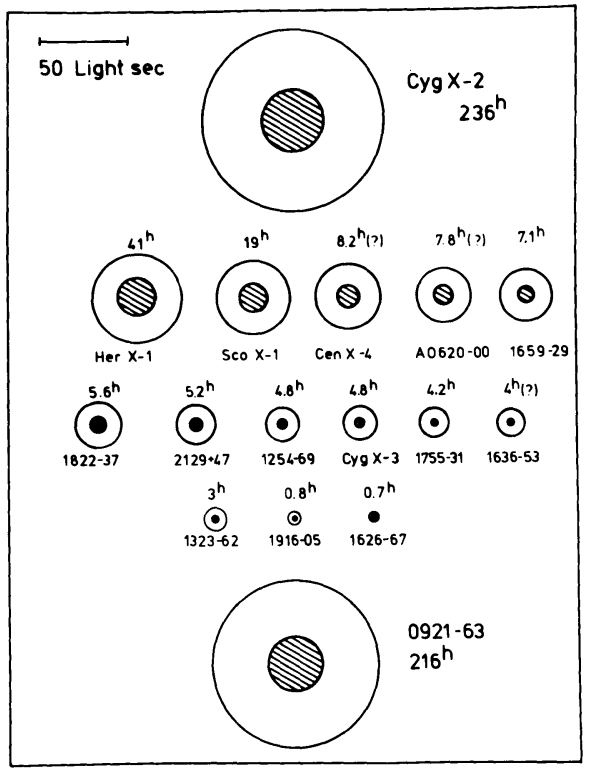

Figure 3. Orbital dimensions and periods of 16 low-mass $X-r a y$ binaries (adapted from McClintock and Rappaport 1984). 
3.4. The formation mechanism of the neutron stars in the two types of binary pulsar systems

In the PSR 1913+16-group the one or two neutron stars present in the system originated by core collapse of a massive star at the end of its nuclear evolution.

On the other hand, for the 1953+29-group and their progenitors, the wide LMXBs, such a scenario seems unlikely for several reasons, notably because in the core collapse of a massive star a considerable amount of mass is being ejected which most probably will have unbound a system in which the companion is a low-mass star (Helfand et al. 1983). A variety of arguments indicate that these neutron stars formed recently, most probably during the accretion process itself, by the accretion-induced collapse of a white dwarf (Taam and Van den Heuvel 1986), abbreviated here as AIC.

The systems with evolved low-mass companions are indeed ideally suited for the occurrence of AIC, since AIC requires a mass-transfer rate $>10^{-8.5}\left(10^{-9}\right) M_{\odot} / y r$. This is because at these accretion rates the accreted hydrogen burns steadily or in weak flashes such that little or no mass is being ejected and the white dwarfs can grow considerably in mass. On the other hand, for $\dot{\mathrm{M}}<10^{-9} \mathrm{M}_{\odot} / \mathrm{yr}$, the accreted matter burns in strong nova-like flashes (Nomoto, this volume), and most or all of the accreted matter is ejected.

Just in the relatively wide systems with evolved low-mass companions a steady high mass transfer rate is maintained (see above and figure $2 \mathrm{~b}$ ), making these systems very suitable for AIC to occur. On the other hand, the short period nova-like binaries in general have much lower transfer rates $\left(\leqslant 10^{-10}-10^{-9} \mathrm{M}_{\odot} / \mathrm{yr}\right)$ and are therefore less well suited for the AIC model. For a more detailed discussion for the conditions of occurrence of AIC we refer to Taam and Van den Heuvel (1986).

3.5. Relation between msec pulsar binaries and QPO sources.

The discovery of QPO $s$ in the two wide LMXBs Cyg X-2 and Sco X-1 as well as in a number of bright galactic bulge $X$-ray sources provides supporting evidence for the wide LMXB-nature of the bright bulge sources (see section 3.3.). The most straightforward interpretation of the QPOphenomenon is provided by the Alpar-Shaham (1985a, b) beat-frequency model (for details: see Shaham, this volume; and Lewin, this volume). This interpretation yields typical rotation periods and magnetic field strengths of the neutron stars in these systems of $\leqslant 10 \mathrm{~ms}$ and $10^{9}$ $10^{10}$ gauss, respectively. These values are very similar to those found in the millisecond binary radio pulsars. Since also the system dimensions of Cyg $\mathrm{X}-2$ and Sco $\mathrm{X}-1$ are similar to those of the progenitors of the PSR 1953+29-group of radio pulsar binaries, the QPOsources seem ideal progenitors for the $1953+29-$ group of radio pulsar binaries. 
3.6. The formation rate of binary millisecond pulsars and the magneticfieldistrength evolution of neutron stars

The total galactic number of bright wide LMXBs is at most some 30 to 50 . Assuming a mass transfer time of $\sim 10^{8}$ yrs (see section 3.3 ) and assuming that half the mass transfer life-time is spent in the precollapse phase, with a white dwarf companion, the galactic formation rate of these systems is $(3-5) \times 10^{-7} \mathrm{yr}^{-1}$. In a steady state this is also the formation rate of the systems of the $1953+29-$ group. This low formation rate provides very strong evidence for the existence of a "bottom" value for the magnetic fields of neutron stars of $\sim 5.10^{8} \mathrm{G}$, below which the fields do not decay (cf. Bhattacharya and Srinivasan 1986; Van den Heuvel et al. 1986).

The reasoning is as follows: if one assumes that neutron-star magnetic fields decay on an $\sim 10^{7} \mathrm{yr}$ timescale, as indicated by radio pulsar observations (cf. Lyne, this volume) then systems of the PSR $1953+29$-group will be observable as radio pulsars for only $\sim 2.10^{7}$ yrs. This is because in order to allow spin up to $\sim 5 \mathrm{msec}$, the magnetic field strength of a neutron star must already be $\leqslant 3.10^{9}$ G. From this value, the field will decay to $\leqslant 5 \cdot 10^{8} \mathrm{G}$ within about two foldings, i.e. $\sim 2.10^{7}$ yrs. Thus, if at $B \leqslant 3.10^{9} \mathrm{G}$ the decay timescale of the magnetic fields of neutron stars is still $\sim 10^{7} \mathrm{yrs}$, one would expect the total number of observable binary millisecond pulsars in the galaxy to be of order $\leqslant 6-10$. However, among the $\sim 500$ known pulsars there are already 2 such systems known. As the distances of these systems are of the same order as those of the bulk of the radio pulsars (namely 0.3 and $3 \mathrm{kpc}$ ) these two systems give a measure for the actual percentage of these systems among the radio pulsars, of order $0.4 \%$, uncorrected for selection effects. With an estimated total galactic number of active pulsars of $(2-5) \times 10^{5}$, the total number of active binary millisecond pulsars becomes $\geqslant 800$ to 2000. Stokes et al. (1986) taking observational selection effects properly into account estimate that the actual galactic number of binary millisecond pulsars is even higher, of order $10^{4}$.

The total number of remnants of LMXBs produced in the entire lifetime of the galaxy $\left(\sim 1.5 \times 10^{10} \mathrm{yr}\right)$ is, for a constant formation rate equal to the present one, of a similar order, i.e. (4.5 - 7.5) $\mathrm{x}$ $10^{3}$. The only way to explain why these numbers are of the same order is that magnetic fields of neutron stars do not decay any more below a value of $\sim(0.5-1) \times 10^{9}$ G. If the magnetic field strengths remain fixed around such a value, the spindown timescale (by magnetic dipole radiation, etc.) is longer than a Hubble time, and all binary millisecond pulsars ever produced in the galaxy will still presently be observable.

This theoretical argument confirms and provides independent support for the earlier findings by Kulkarni (1986a, b) indicating that the cooling age of the white dwarf optical companion of PSR 0655+64 is $\geqslant$ $(0.5) \times 10^{9} \mathrm{yrs}$. Since the field strength of PSR $0655+46$ is sti11 $\sim 10^{10}$ $G$ it seems that in some neutron stars the field decay may already stop at this value. 
3.7. Possible interpretations for the existence of a "bottom field strength" in neutron stars

Two interpretations seem possible, namely:

A. In terms of the Flowers and Ruderman (1977) model. In this model the magnetic field of a neutron star consists of two parts: (i) the strong observable outside field of $\sim 10^{12-13} \mathrm{G}$ in young pulsars, which is anchored in the crust, and supported by crustal electric currents. These currents resulted from flux-freezing and dynamo action shortly before and during the collapse, and (ii) a field seated in the superconducting interior.

The crustal part of the field will, in this model, decay on a relatively short timescale $\sim 10^{7} \mathrm{yrs}$, due to ohmic dissipation of the crustal electric currents.

On the other hand, the field in the superconducting interior will never decay.

In terms of this model, the final outside field of $\sim 5.10^{8} \mathrm{G}$ is connected with superconducting interior and will remain forever.

B. Accretion-induced decay, as suggested by Taam and Van den Heuvel (1986), as an alternative interpretation of the observations. In this semi-empirical "model", magnetic fields of non-accreting neutron stars may not decay at all (cf. Kundt 1981), but accretion of matter would gradually decrease the strength of the exterior dipole field.

All our knowledge about magnetic fields of neutron stars in Xray binaries and radio pulsar binaries is consistent with such an interpretation (Taam and Van den Heuvel 1986). Those neutron stars that have the weakest fields (the binary millisecond pulsars) are indeed just the ones that underwent the largest amount of accretion, and vice versa. (Of course, this inverse correlation can also be seen as an inverse correlation between magnetic field strenth and time, i.e.: as just another manifestation of the spontaneous decay of neutron star magnetic fields in general. This is, because the amount of matter accreted is, roughly, proportional to time, as the accretion rate is, - within an order of magnitude - equal to the Eddington limit in most systems).

A possible theoretical model in which the field strength can be reduced by accretion has been suggested by Blondin and Freese (1986).

\section{THE FORMATION OF THE SINGLE MILLISECOND PULSAR PSR $1937+21$}

The location of this pulsar in the B vs. $P$ diagram (figure 1) close to the spin-up line, suggests strongly that it was also recycled in a binary system (Radhakrishnan and Srinivasan 1982; Alpar et al. 1982). In this case the companion must have been lost. This can have happened in two ways, either (i) by a supernova explosion of the companion which caused the system to become unbound, or (ii) by coalescence with the companion, in a close system in which the orbital angular momentum was lost, for example, by GR-losses.

The first possibility seems ruled out (see Henrichs and van den Heuvel 1983; Van den Heuvel 1986b). Thus, the only binary models which seem to 
remain are the coalescence models, which we will now briefly discuss. Three types of coalescence models have been proposed which all seem

- in principle - feasible, namely:

(i) coalescence with a second neutron star (Henrichs and van den Heuvel 1983);

(ii) coalescence with a massive white dwarf ( $>0.65 \mathrm{M}_{\odot}$ ) (Van den Heuvel and Bonsema 1984; Bonsema and van den Heuve1 1985).

(iii) coalescence with a low-mass degenerate dwarf, either hydrogen- or helium rich (Alpar et. al. 1982; Ruderman and Shaham 1983, 1985; Fabian et al. 1983).

For each of these models examples of progenitor systems are known, viz.:

(i) PSR 1913+16, consists of two neutron stars, which will certainly coalesce in the future, since the observed timescale of orbital decay by GR losses is $3.10^{8}$ yrs (Weisberg and Taylor 1984).

(ii) PSR $0655+64$ consists of a neutron star and a massive white dwarf. If such a system were born with a slightly shorter orbital period, $<12^{\mathrm{h}}$ (which is certainly expected in some cases of spiral-in as depicted in figure 2a), then coalescence due to GR losses will occur within a Hubble time.

(iii) Systems with a red dwarf companion are the LMXBs with short orbital periods, less than $\sim 8$ (see figure 3 ).

The advantages and disadvantages of each of these three models are extensively discussed elsewhere (Van den Heuvel 1986b) to which we refer for details. The third model is possible only if large angular momentum losses from the system are invoked, to a rather unlikely degree (see Taam and Wade 1985). Furthermore, at the time of coalescence the masstransfer rate in these systems has become so low that the neutron star will have been spun down to a long period, of the order of $\sim 0.1$ second. During the coalescence the amount of mass accreted $\left(\leqslant 0.005 \mathrm{M}_{\odot}\right)$ is insufficient to spin the neutron star back up to a millisecond period (Jeffrey 1985). For these reasons the other two coalescence models seem preferrable for the origin of PSR $1937+21$. The progenitor systems in both these models were massive binary systems (see figure $2 b$ ), contrary to the progenitors of the two binary millisecond pulsars.

REFERENCES

Alpar, M.A., Cheng, A.F., Ruderman, M.A. and Shaham, J., 1982, Nature $300,728$.

Alpar, M.A. and Shaham, J., 1985a, IAU Circ. No.4046.

Alpar, M.A. and Shaham, J., 1985b, Nature 316, 239.

Arons, J., 1981, IAU Symp. No.95, 69 .

Blaauw, A., 1961, Bull. Astron. Inst. Netherlands 15, 265.

Bhattacharya, D. and Srinivasan, G., 1986, Current Science 55, 327.

Bisnovaty-Kogan, G.S. and Komberg, B.V., 1974, Astron.Zh. 51, 373

(Soviet Astron. 18, 217).

Blondin, J.M. and Freese, K., 1986, Nature (in the press).

Bodenheimer, P. and Taam, R.E., 1984, Astrophys. J. 280, 771.

Bonsema, P.F.J. and van den Heuvel, E.P.J., 1985, Astr. Astrophys. 146, L3.

Fabian, A.C., Pringle, J.E., Verbunt, F., Wade, R., 1983, Nature 301, 222 .

Flowers, E. and Ruderman, M.A., 1977, Astrophys. J. 215, 302. 
Habets, G.M.H.J., 1985, Ph.D. Thesis, University of Amsterdam. Habets, G.M.H.J., 1986, Astr. Astrophys. (in the press). Helfland, D.J., Ruderman, M.A. and Shaham, J., 1983, Nature 304, 423. Henrichs, H.F., 1983, in: Accretion-Driven Stellar X-ray Sources, W.H.G.

Lewin and E.P.J. van den Heuvel (eds.), Cambridge Univ. Press, 393. Henrichs, H.F. and van den Heuvel, E.P.J., 1983, Nature 303, 213. Jeffrey, L.C., 1985, Nature 319, 384. Joss, P.C. and Rappaport, S.A., 1983, Nature 304, 419. Kulkarni, S.R., 1986a, IAU Circ. No.4160. Kulkarni, S.R., 1986b, Astrophys. J. (in the press). Kundt, W., 1981, Astr. Astrophys 98, 207. McClintock, J.E. and Rappaport, S.A. Proc. Seventh North American Workshop on Cataclysmic Binaries and Low-Mass X-ray Binaries Center for Astrophys.). In the press.

Meyer, F. and Meyer-Hofmeister, E., 1979, Astr. Astrophys. 78, 167. Paczynski, B., 1971, Ann. Rev. Astr. Astrophys. 9, 183.

Radhakrishnan, V., 1982, Contemp. Phys. 23, 207.

Radhakrishnan, V., 1986, in: Highlights of Astronomy 7, Reide1. Pub. Comp. Dordrecht.

Radhakrishnan, V. and Srinivasan, G., 1982, Curr. Science 51, 1096. Rappaport, S.A. and Joss, P.C., 1983, in: Accretion-Driven Stellar X-ray Sources, Eds. W.H.G. Lewin and E.P.J. van den Heuve1, Cambridge Univ. Press, 1.

Rappaport, S.A., Verbunt, F. and Joss, P.C., 1983, Astrophys. J. 275, 713.

Ruderman, M.A. and Shaham, J., 1983, Nature 304, 425. Ruderman, M.A. and Shaham, J., 1985, Astrophys. J. 289, 244. Ruderman, M.A. and Sutherland, P.G., 1976, Astrophys. J. 196, 51. Smarr, L.L. and Blandford, R.D., 1976, Astrophys.J. 207, 574. Stokes, G.H., Segelstein, D.J., Taylor, J.H. and Dewey, R.J., 1986, Astrophys. J. (in the press).

Taam, R.E., 1983, Astrophys. J. 270, 694.

Taam, R.E., Bodenheimer, P., Ostriker, J.P., 1978 Astrophys. J. 222, 269.

Taam, R.E., and van den Heuvel, E.P.J., 1986, Astrophys. J. 305, 235. Taam, R.E. and Wade, R.A., 1985, Astrophys. J. 293, 504 . van den Heuvel, E.P.J., 1984, J. Astrophys.Astr. 5, 209. van den Heuvel, E.P.J., 1986a, in: The Evolution of Galactic X-ray Binaries, eds. J. Truemper et alı, Reidel Pub. Comp. Dordrecht, 107. van den Heuve1, E.P.J., 1986b, in: High-Energy Phenomena Around Collapsed Stars, Ed. F. Pacini, Reidel Pub. Comp, Dordrecht (in the press). van den Heuve1, E.P.J. and Bonsema, P.F.J., 1984, Astr. Astrophys. 139, L16.

van den Heuvel, E.P.J. and Habets, G.M.H.J., 1985, in: Supernovae, Their Progenitors and Remnants, Eds. G. Srinivasan and V. Radhakrishnan, Indian Acad. Sc., Bangalore, 129.

van den Heuvel, E.P.J. and Taam, R.E., 1984, Nature 309, 235.

van den Heuvel, E.P.J., van Paradijs, J.A. and Taam, R.E., 1986, Nature (in the press).

Webbink, R.F., Rappaport, S.A. and Savonije, G.J., 1983, Astrophys.J. 270,678 .

Weisberg, J.M. and Taylor, J.H., 1984, Phys. Rev• Letters 52, 1348. 


\section{DISCUSSION}

D. Arnett: Have you considered the effects on the companion of pushing a white dwarf over the Chandrasekhar limit?

B. van den Heuve1: Taam and I have looked into this problem. If the ejected amount of mass is not more than a few tenths of a solar mass, we find, using the results of Fryxell and Arnett, that the effects on a red dwarf companion are negligible, although the system gets a runaway velocity due to the impact. In the case of a giant companion, where the envelope is less strongly bound, the effects might be stronger.

G. Hasinger: If you do not believe in field decay, do you still need the accretion-induced collapse of a white dwarf?

E. van den Heuve1: If one does not believe in field decay, my reasoning that PSR $0820+02$ must be young will no longer hold and accretion-induced collapse (AIC) is no longer required to explain its strong field. There are, however, additional reasons which make AIC likely for these systems, namely that the mass loss associated with the core collapse of a massive star would have unbound a system with a low-mass companion, as was pointed out by Helf and et al. (1983).

J.H. Huang: It may be worthwhile to point out that the neutron star formed by accretion-induced collapse in a binary will, after the collapse, continue to accrete. So, it may grow to a mass of up to 2 solar masses (by accreting for $10^{8}$ yrs in a low-mass $X$-ray binary). I could imagine that such neutron stars have a completely different interior structure than neutron stars formed in a type II supernova. This might also be a reason for finding two types of pulsars.

N. White: Could you push the neutron star over its maximum mass into a black hole?

E. van den Heuvel: In principle this is a viable possibility in the low-mass systems, where the neutron star may accrete as much as a solar mass. If the upper mass limit for neutron stars is somewhere near 2.0 to 2.2 solar masses, as preferred equations of state seem to indicate, this might indeed happen.

J. Shaham: Let's go back for a moment to the "0.005 $\mathrm{M}_{\odot}$ " scenario you mentioned earlier. What happens to a system of a neutron star and a $0.4 \mathrm{M}_{\ominus}$ main sequence star, say, that is driven by gravitational radiation, as it evolves? According to the Jeffery paper, it spends most of its life at rates of $10^{-12} \mathrm{Mo} /$ year or less. However - one does not see many systems like that among the LMXBs. That may indicate, that either some process prevents $M$ from getting that small (onset of large mass and angular momentum loss from the system, for example, which speeds up the evolution) or that the starting binary does not exist. What is your view on that?

B. van den Heuve1: This is an important point, to which I do not have a ready answer. We indeed do not know many systems with $\mathrm{X}-\mathrm{ray}$ luminosities between $10^{33}$ and $10^{35}$ ergs $\mathrm{s}^{-1}$, although according to the models you mentioned, many such sources would be expected to exist. A possible answer might be that once the mass 
tranfer rate drops below $10^{-10} \mathrm{M}_{\odot} / \mathrm{yr}$, the sources become transients instead of steady sources. We do indeed observe quite a number of soft X-ray transients, and their total number may be very large. Verbunt has in fact, in his talk here, suggested that the many weak sources seen in globular clusters are such transients in their quiescent state.

R. Manchester: Would you expect the process of coalescence in the formation of PSRl937+21 to cause the period to depart from the equilibrium spin-up value?

E. van den Heuve1: In the red-dwarf case this is indeed expected, as was shown by Jeffrey (Nature 319, 384-386, 1986): she expects that the neutron star then settles at the equilibrium period corresponding to the final mass transfer rate of $\lesssim 10^{-12} \mathrm{M} \odot \mathrm{yr}$, which is of the order of one second. Also in the case of coalescence with a massive white dwarf it is not clear whether it will settle near the spin-up line. If super-Eddington accretion would occur - since the mass transfer is highly super-Eddington - it might end up to the left of the spin-up line. The fact that it is just located on this line may, however, well indicate that super-Eddington accretion cannot occur over long intervals of time. In the case of coalescence of two neutron stars the endproduct has a rotation period of $1.5 \mathrm{msec}$ which, by coincidence, just happens to fall on the spin-up line for the present field strength of the millisecond pulsar.

S. Kulkarni: The model you describe for low-mass radio binary pulsars will fail when $\mathrm{P}_{0}$, the initial orbital period is less 10 days since the accretion rate on the white dwarf will then be less that $10^{-9} \mathrm{M}_{\ominus} / \mathrm{yr}$ and hence does not result in an accretion induced neutron star. The existence of LMXBs with present periods less than 10 days (ex. 4U1626-67 and 1E2259+586) and the low $z$-distribution of low-mass binary pulsars suggest that, in fact, the radio binary pulsars come from Population I (or intermediate population) stars. Could you please comment on this radical proposa1?

B. van den Heuvel: The formula for the evolution-driven accretion rate which $\mathrm{I}$ used yields $\dot{\mathrm{M}}<10^{-9} \mathrm{M}_{\Theta} / \mathrm{yr}$ only for $\mathrm{P} \lesssim 2$ days. However, for just these short periods the formula (which is an approximation, and moreover, also depends on the heavy-element abundance) no longer yields the correct mass-transfer rate, since for these short periods $\left(\leqslant 4^{\mathrm{d}}\right)$ the effects of angular momentum loss by magnetic braking also become important. In fact, the effects of magnetic braking increase towards the shorter periods, causing the mass-transfer rate to remain $\gtrsim 10^{-9} \mathrm{M} / \mathrm{yr}$ in the period range of $\sim 0.5$ to $>2$. So even for LMXBs and binary pulsars with orbital periods in this range, the neutron stars may still have formed by accretion-induced collapse. For the shorterperiod systems, such as 4U1626-67 this may still be the case, provided that the companion was a hydrogen-poor star (cf. Taam and van den Heuvel 1986; Savonye, de Kool and van den Heuvel 1986). 